\title{
A Hybrid Numerical Technique for the Solution of a Class of Implicit Matrix Differential Equation
}

\author{
Nicoletta Del Buono and Luciano Lopez \\ Dipartimento di Matematica, Università degli Studi di Bari, \\ Via E. Orabona, 4, I-70125 Bari, ITALY [delbuono, lopezl]@dm.uniba.it
}

\begin{abstract}
This paper is concerned with the numerical solution of an implicit matrix differential system of the form $Y^{T} \dot{Y}-F(t, Y)=0$, where $Y(t)$ is a $n \times n$ real matrix which may converge to a singular matrix. We propose a hybrid numerical technique based on an implicit second order Runge Kutta scheme which derives a particular algebraic Riccati equation and via its solution approximates the solutions of the differential problem at hand. Numerical examples demonstrating the behavior of the proposed approach are also reported.
\end{abstract}

\section{Introduction}

Many physical and industrial processes are most naturally and easily modeled as sets of implicit differential equations (IDEs) or as mixed systems of differential and algebraic equations (DAEs) and in the last decades there has been an increasing interest in exploiting the advantages of working directly with these implicit models ([2], 5], [1] $)$. In this paper we concentrate our attention on problems whose general form is that of an implicit differential equation

$$
G(t, Y(t), \dot{Y}(t))=Y^{T}(t) \dot{Y}(t)-F(t, Y(t))=0, \quad t \geq t_{0},
$$

where $G$ and $Y$ are square real matrices of the same dimension $(n \times n)$ and $G$ is supposed to have sufficiently many bounded derivatives (i.e. $F$ is assumed to be a sufficiently smooth matrix function mapping $\mathbb{R} \times \mathbb{R}^{n \times n}$ into $\left.\mathbb{R}^{n \times n}\right)$. The initial value $Y\left(t_{0}\right)$ is supposed to be specified and the solution $Y(t)$ is sought on a bounded interval $\left[t_{0}, T\right]$.

If the partial derivative of $G$ with respect to $\dot{Y}$ is invertible for all $t \in\left[t_{0}, T\right]$ then we can formally solve $\dot{Y}$ in (II) to obtain a set of ordinary differential equations:

$$
\dot{Y}(t)=Y^{-T}(t) F\left(t, Y(t), \quad Y\left(t_{0}\right)=Y_{0} .\right.
$$

Examples of the above ODEs appear in some matrix inverse eigenvalue problems, [6], feedback control problems [12, in the context of geometric integration on matrix manifolds [8], in multivariate data analysis [17]. 
Here we are interested in the case when $\frac{\partial G}{\partial \dot{Y}}=Y^{T}$ crosses a singular matrix at some instant $\tau \leq T$. In fact it has been proved in [7] that the property of the solution $Y(t)$ to be non singular for all $t \in\left[t_{0}, T\right]$ is not guaranteed a priori since the presence of a finite escape point $\tau \leq T$, where $Y(\tau)$ may become singular, is not precluded.

Numerical approaches for the solution of the problem at hand, must therefore take into account this behavior and may be divided roughly into two classes: direct discretization of the given system and methods which involve a reformulation combined with a discretization.

The desire for as direct a discretization as possible arises because a reformulation may be costly, may require additional input (enlarging the dimension of the problem) and may be more cumbersome than the original problem. In fact, generally, any implicit differential equations of the form (11) can be written in a semi-explicit form upon defining $\dot{Y}=Z$, which leads to

$$
\begin{aligned}
& \dot{Y}=Z \\
& 0=G(t, Y, Z)=Y^{T} Z-F(t, Y) .
\end{aligned}
$$

The latter differential algebraic equation with constraint may be solved carrying out a regularization technique, that is replacing the algebraic constraint by an ordinary differential equation depending on a small parameter $0 \leq \varepsilon \ll 1$ to get then

$$
\begin{aligned}
& \dot{Y}=Z \\
& \varepsilon \dot{Z}=G(t, Y, Z) .
\end{aligned}
$$

The ordinary differential system derived from this regularization techniques is very stiff (see for instance [2] and [5]), and its numerical solution is typically more arduous. Moreover, as observed in [7], the solution $Y(t)$ can present an additional structure to be preserved during the integration, which might be destroyed by regularization. Hence, the above discussion justifies the research of a direct discretization of (11).

In the remainder of the paper we detail how a direct discretization formula is carried out to derive from (1) a general Riccati equation which can be easily solved. The numerical treatment of this Riccati equation is also discussed. Finally, some numerical tests illustrate the behavior of the proposed hybrid approach and some concluding remarks are sketched.

\section{Hybrid Numerical Technique}

As observed before, when the solution of (1) approaches a singular matrix at some isolated point $\tau$, the Jacobian matrix of (1) becomes singular too. In this case, since the explicit ordinary differential equation (2) cannot be derived, a direct discretization of $G(t, Y(t), \dot{Y}(t))=0$ have to be considered approximating $Y(t)$ and $\dot{Y}(t)$, for instance, by a Runge Kutta scheme.

Runge Kutta methods have been originally conceived for numerical solution of ordinary differential equations. From an approximation $Y_{n}$ of the solution at 
the instant $t_{n}$, they construct an approximation of $Y_{n+1}$ at $t_{n+1}=t_{n}+h$, where $h$ is a constant step-size, via the formula:

$$
Y_{n+1}=Y_{n}+h \sum_{i=1}^{s} b_{i} \dot{Y}_{n i}
$$

where $\dot{Y}_{n i}$ is explicitely defined as a function of the internal stages $Y_{n i}$ given by:

$$
Y_{n i}=Y_{n}+h \sum_{i=1}^{s} a_{i j} \dot{Y}_{n i}, \quad i=1, \ldots, s,
$$

where $a_{i j}, b_{i}, c_{i}$ are the coefficients defining the specific method and $s$ is the number of the stages. To extend Runge Kutta method to implicit differential equation $G(t, Y, \dot{Y})=0$ we define $Y_{n+1}$ as the solution of (3), (4) and

$$
G\left(t_{n}+c_{i} h, Y_{n i}, \dot{Y}_{n i}\right)=0
$$

Explicit Runge Kutta methods are not directly suitable for the implicit equation in interest because, at each step, $Y_{n+1}$ have to be determined as the solution of a linear system which can become very ill conditioned.

This can be explicitely observed applying, for instance, the forward Euler method to (1), that is

$$
G\left(t_{n}, Y_{n}, \frac{Y_{n+1}-Y_{n}}{h}\right)=0 .
$$

This provides the following system of linear equation for $Y_{n+1}$

$$
Y_{n}^{T} Y_{n+1}-Y_{n}^{T} Y_{n}-h F\left(t_{n}, Y_{n}\right)=0 .
$$

Unfortunately this simple method does not work when the numerical solution is approximating a singular matrix (i.e. $\operatorname{det}\left(Y_{n}\right) \simeq 0$ ), therefore stability considerations indicate an implicit discretization rule as the most suitable to be used.

An interesting result can be obtained applying to (1) the second order Gauss Legendre Runge Kutta scheme defined by the Butcher array

$$
\begin{array}{l|c}
1 / 2 & 1 / 2 \\
\hline & 1
\end{array}
$$

which applied to the implicit differential equation (1) provides

$$
Y_{n+1}^{T} Y_{n+1}+Y_{n}^{T} Y_{n+1}-Y_{n+1}^{T} Y_{n}-Y_{n}^{T} Y_{n}-2 h F\left(t_{n}+\frac{h}{2}, \frac{Y_{n}+Y_{n+1}}{2}\right)=0 .
$$

In the following we will show how to solve the nonlinear equation (6) in order to get at each step a numerical approximation of $Y\left(t_{n+1}\right)$. 


\subsection{On the Solution of the Algebraic Riccati Equation}

To avoid the nonlinearity embedded in $F$, we solve recursively equation (6) starting from a guess approximation $Y_{n+1}^{(0)}$, which can be chosen equals to $Y_{n}$, that is we resolve:

$$
Y_{n+1}^{T} Y_{n+1}+Y_{n}^{T} Y_{n+1}-Y_{n+1}^{T} Y_{n}-Y_{n}^{T} Y_{n}-2 h F\left(t_{n}+\frac{h}{2}, \frac{Y_{n}+Y_{n+1}^{(0)}}{2}\right)=0 .
$$

Setting $A=Y_{n}$ and $C=\left(Y_{n}^{T} Y_{n}+2 h F\left(t_{n}+\frac{h}{2}, \frac{Y_{n}+Y_{n+1}^{(0)}}{2}\right)\right)$ the latter equation reads as a general algebraic Riccati equation

$$
\mathcal{R}(X)=X^{T} X+A^{T} X-X^{T} A+C=0 .
$$

Algebraic Riccati equation have been largely treated ([1], 10], 15]).We will assume that (7) possesses a unique solution $X \in \mathbb{R}^{n \times n}$.

To approximate the solution of the Riccati equation we apply the Newton iteration. The Riccati function $\mathcal{R}$ is clearly a mapping from $\mathbb{R}^{n \times n}$ into itself. Moreover, the first Frechet derivative of $\mathcal{R}$ at a matrix $X$ is the linear map $\mathcal{R}_{X}^{\prime}: \mathbb{R}^{n \times n} \rightarrow \mathbb{R}^{n \times n}$, which applied to $H \in \mathbb{R}^{n \times n}$ is given by

$$
\mathcal{R}_{X}^{\prime}(H)=H^{T} X+X^{T} H+A^{T} H-H^{T} A=H^{T}(X-A)+(X+A)^{T} H .
$$

Then the Newton iteration for (8) is as follows:

- starting from $X_{0}$

- the solution of $\mathcal{R}(X)=0$ can be obtained recursively as

$$
X_{j+1}=X_{j}+D_{j}
$$

with $D_{j}$ solution of the Sylvester equation

$$
\mathcal{R}_{X}^{\prime}\left(D_{j}\right)=-\mathcal{R}\left(X_{j}\right) \Leftrightarrow\left(X_{j}+A\right)^{T} D_{j}+D_{j}^{T}\left(X_{j}-A\right)=-\mathcal{R}\left(X_{j}\right) .
$$

It appears clear that at each step we have to focus on the numerical treatment of the linear matrix equation

$$
\mathcal{A} X+X^{T} \mathcal{B}=\mathcal{C}
$$

with $\mathcal{A}, \mathcal{B}, \mathcal{C} \in \mathbb{R}^{n \times n}$ given matrices. The first question arising in examinating the above linear equation is about its solvability. An answer to this question can be found in [16] (see also [3], 14]); we report it in the following.

Theorem 1. Let $\mathcal{A}, \mathcal{B}, \mathcal{C} \in \mathbb{R}^{n \times n}$; there exists some matrix $X \in \mathbb{R}^{n \times n}$ such that $\mathcal{A} X+X^{T} \mathcal{B}=\mathcal{C}$ if and only if

$$
\operatorname{rank}\left[\begin{array}{ll}
\mathcal{C} & \mathcal{A} \\
\mathcal{B} & O
\end{array}\right]=\operatorname{rank}\left[\begin{array}{ll}
O & \mathcal{A} \\
\mathcal{B} & O
\end{array}\right] .
$$

(Note that this condition is equivalent to ask that there exist nonsingular matrices $P \in \mathbb{R}^{m \times m}$ and $Q \in \mathbb{R}^{n \times n}$ such that

$$
P\left[\begin{array}{ll}
\mathcal{C} & \mathcal{A} \\
\mathcal{B} & O
\end{array}\right] Q=\left[\begin{array}{ll}
O & \mathcal{A} \\
\mathcal{B} & O
\end{array}\right] .
$$


Conditions on the uniqueness of the solution of (10) and on the construction of such a solution $X$ can be obtained rewriting (10) as an ordinary linear system of $n^{2}$ equations in $n^{2}$ unknowns, using the Kronecker product $\otimes$ and the function vec from the space of $n \times n$ matrices to the space of $n^{2}$ vectors.

In fact, (10) is equivalent to:

$$
(I \otimes \mathcal{A}) \operatorname{vec}(X)+\left(\mathcal{B}^{T} \otimes I\right) \operatorname{vec}\left(X^{T}\right)=\operatorname{vec}(\mathcal{C}) .
$$

Note that there exists a permutation matrix (see [14]) which maps the vector $\operatorname{vec}\left(X^{T}\right)$ into $\operatorname{vec}(\mathrm{X})$, that is:

$$
\operatorname{vec}\left(X^{T}\right)=P(n, n) \operatorname{vec}(X)
$$

being $P(n, n)$ the permutation matrix such that

$$
P(n, n)=\sum_{i=1}^{n} \sum_{j=1}^{n} E_{i j} \otimes E_{i j}^{T}
$$

where $E_{i j} \in \mathbb{R}^{n \times n}$ with elements 1 in the $i j$ position and zeros elsewhere. Hence, rearranging (11) we obtain

$$
\left[(I \otimes \mathcal{A})+\left(\mathcal{B}^{T} \otimes I\right) P(n, n)\right] \operatorname{vec}(X)=\operatorname{vec}(\mathcal{C}) .
$$

Hence it follows immediately that the solution $X$ of 10 exists and is unique if the coefficient matrix $\left[(I \otimes \mathcal{A})+\left(\mathcal{B}^{T} \otimes I\right) P(n, n)\right]$ has full rank $n^{2}$.

It should be pointed out that using the Newton's method (9), the coefficient matrix is of full rank and therefore a unique solution is ensured. About the convergence of the Newton's procedure, since the starting matrix for the iteration is an approximation of the solution of the differential system at a previous step, with an appropriate choice of the step size $h$, the algorithm converges in a reasonable number of iteration.

The linear system involved in the solution of the algebraic Riccati equation can be solved using direct methods such as Gaussian elimination or QRdecomposition or, when the dimension increases, any iterative solver which preserves also the sparsity of the coefficient matrix. Equation (10) can be also solve applying a subspace method as suggested in [4].

The following algorithm summerizes the complete hybrid scheme described before for the solution of the implicit differential equation (11):

\section{GL2Riccati method}

Given a step size $h>0$, an approximation $Y_{n}$ of $Y\left(t_{n}\right)$, and a user defined tolerance tol, compute an approximation $Y_{n+1}$ of the solution $Y\left(t_{n}+h\right)$ as follows:

(1) Set $k=0$ and $Y_{n+1}^{(k)}=Y_{n}$;

(2) set $A=Y_{n}$ and compute $C=Y_{n}^{T} Y_{n}+2 h F\left(t_{n}+\frac{h}{2}, \frac{Y_{n}+Y_{n+1}^{(k)}}{2}\right)$

(3) apply the Newton method to the Riccati equation

$$
\mathcal{R}(X)=X^{T} X+A^{T} X-X^{T} A+C=0
$$


(3a) Set $j=0$ and $X_{j}=Y_{n}$

(3b) Compute the solution $D_{j}$ of the equation

$$
\left(X_{j}+A\right)^{T} D_{j}+D_{j}^{T}\left(X_{j}+A\right)=-\mathcal{R}\left(X_{j}\right)
$$

Use a direct or an iterative algorithm for linear system (12).

(3c) Compute $X_{j+1}=X_{j}+D_{j}$

(4) Set $j=j+1$ and iterate until $\left\|\mathcal{R}\left(X_{j+1}\right)\right\| \leq$ tol

(5) Set $k=k+1$ and $Y_{n+1}=Y_{n+1}^{(k)}=X_{j}$

(6) Repeat from (2) until:

$$
\left\|Y_{n+1}^{T} Y_{n+1}-A Y_{n+1}^{T}-A^{T} Y_{n+1}-Y_{n}^{T} Y_{n}-2 h F\left(t_{n}+\frac{h}{2}, \frac{Y_{n}+Y_{n+1}^{(k)}}{2}\right)\right\| \leq \text { tol }
$$

\section{$3 \quad$ Numerical Tests}

In this section we report some numerical tests in order to illustrate the behavior of the proposed approach. All the numerical results have been obtained by Matlab 6.1 codes implemented on Pentium IV 1.6GHz with 1GbRAM. We compare the GL2Riccati scheme with SVDRK2, the second order Runge Kutta scheme applied to the singular value decomposition approach illustrated in [7] and [9].

\section{$3.1 \quad$ Example 1}

As first example we consider the differential system

$$
Y^{T} Y^{\prime}-\left(\begin{array}{cc}
0 & -\frac{\delta}{2} \\
-\frac{\delta}{2} & 0
\end{array}\right)=0, \quad Y(0)=\frac{1}{\sqrt{2}}\left(\begin{array}{cc}
1 & -1 \\
1 & 1
\end{array}\right)
$$

with $\delta \neq 0$, which has solution given by

$$
Y(t)=\frac{1}{\sqrt{2}}\left(\begin{array}{cc}
\sqrt{1+\delta t}-\sqrt{1+\delta t} \\
\sqrt{1-\delta t} & \sqrt{1-\delta t}
\end{array}\right)
$$

existing in the neighborhood $(-1 / \delta, 1 / \delta)$ of $t_{0}=0$. In this case the matrix function $G(Y)$ exists and is a continuous and Lipschitz matrix function only in a neighborhood of $Y(0)$. We solve a problem with $\delta=1 / 2$. Figure 1 depicts the behavior of the global error on the interval [1,2] of GL2Riccati scheme (solid line) and SVDRK2 (dotted line). The two methods have been applied with the same step size and show the same performance inside the chosen interval.

\subsection{Example 2}

As second example we consider the differential system

$$
Y^{T} \dot{Y}-\left(\begin{array}{cc}
-\sin (t) \cos (t) & \cos (t) \\
-t \sin (t) & t
\end{array}\right)=0
$$

whose matrix solution 


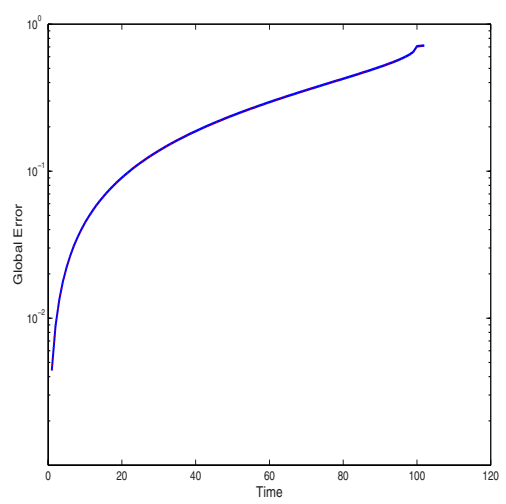

Fig. 1. Behavior of the global error

$$
Y(t)=\left(\begin{array}{cc}
\cos (t) & t \\
0 & 1
\end{array}\right)
$$

defined for all $t$, but periodically, at each multiple of the point $\tau_{k}=\pi / 2$ becomes a singular matrix. We integrate this system in the interval $[\pi / 4, \pi / 2]$, with a step size $h=0.01$. Figure 2 provides the semilog plot of the global error on the integration interval of GL2Riccati scheme (solid line) and SVDRK2 (dotted line). In this case the GL2Riccati approach shows a reduced global error with respect that of SVDRK2, moreover while this latter method blows up at the singularity, the proposed procedure is able to integrate the system at the singularity even if an order reduction can occur.

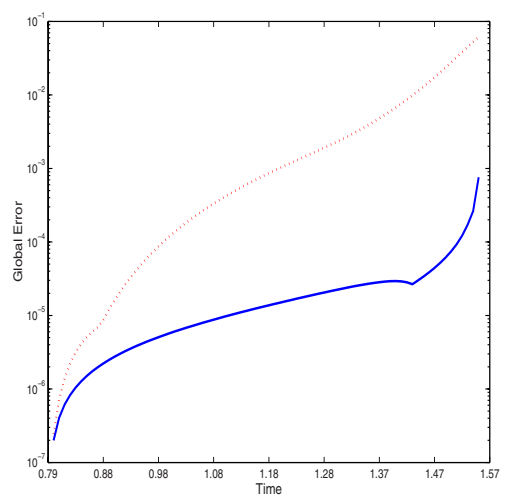

Fig. 2. Behavior of the global error 


\section{Conclusion}

Implicit differential equations play a key role in many applications. In this paper we have considered a particular class of IDE whose solution matrix not guaranteed to be nonsingular for all $t$. We have demonstrated the relationship between the numerical solution of this class of IDE and a general Riccati equation, suggesting a hybrid techniques which provides a numerical approximation of the solution also at escape points, moreover it should be highlighted that since the proposed procedure is based on a second order Gauss Legendre Runge Kutta scheme it is able to preserve any eventual quadratic structure of the theoretical solution.

\section{References}

1. Ammar G., Benner P., and Mehrmann V., A multishift algorithm for the numerical solution of algebraic Riccati equations, ETNA 1 (1993) 33-48.

2. Ascher U.M., Petzold L.R., Computer methods for Ordinary Differential Equations and Differential-Algebraic Equations SIAM 1998.

3. Baksalary J.K., Kala R., The matrix equation $A X-Y B=C$. Linear Algebra Appl. 25 (1979) 41-43.

4. Brands J., Computing tall skinny solutions of $A X-X B=C$. Mathematics and Computers in Simulation 61 (2003) 385-397.

5. Brenan W.E., Campbell S.L., Petzold L.R., Numerical Solution of Inital-value Problems in Differential Algebraic Equations. North-Holland: New York, 1989.

6. Chu, M.T., Inverse Eigenvalue Problems, SIAM Review 40 (1998) 1-39.

7. Del Buono N., Lopez L., Numerical integration of a class of ordinary differential equations on the general linear group of matrices. Numerical Algorithms 34, (2003) 271-282.

8. Del Buono N., Lopez L., Geometric integration on manifold of square oblique rotation matrices. SIAM J. Matrix Anal. Appl. 23(4) (2002) 974-989.

9. Del Buono N., Lopez L., Numerical solution of matrix ordinary differential equations with singular solutions. Tech. Report, Department of Mathematics, University of Bari, Italy, (2004).

10. Guo C.H., Laub A.J., On a Newton-like method for solving algebraic Riccati equations. SIAM J. Matrix Anal. Appl. 21(2) (2000) 694-698.

11. Hairer E., Lubich C., Roche M., The numerical solution of differential algebraic systems by Runge Kutta methods, Lecture Notes in Mathematics, 1409, SpringerVerlag 1989

12. Helmke U., Moore J.B., Optimization and dynamical systems. Springer-Verlag: London, 1994.

13. Horn R.A., Johnson, C.A., Matrix Analysis. Cambridge University Press: New York, 1985.

14. Horn R.A., Johnson, C.A., Topics in Matrix Analysis. Cambridge University Press: New York, 1991.

15. Lancaster P., Rodman L., Algebraic Riccati Equations. Clarenton Press: Oxford, 1995.

16. Roth W.E., The equation $A X-Y B=C$ and $A X-X B=C$ in matrices. Proc. Am. Soc. 3, (1952) 392-396.

17. Trendafilov N.T., Lippert R.A.,The Multi-Mode Procrustes problem. Linear Algebra and Applications 349(1-3), (2002) 245-264. 